\title{
Chaotic Behavior in a Simple DC Drive
}

\author{
K.T. Chau, J.H. Chen, C.C. Chan, Jenny K.H. Pong, and D.T.W. Chan* \\ Department of Electrical \& Electronic Engineering, The University of Hong Kong, Pokfulam Road, Hong Kong \\ * Department of Electrical \& Communications Engineering, Hong Kong Technical College, Tsing Yi, Hong Kong
}

\begin{abstract}
Remarkably complex behavior, namely chaotic behavior, in a simple dc chopper-fed dc motor drive system has been investigated. An iterative map that describes the nonlinear dynamics of the system operating in the continuous conduction mode is derived. It shows that different bifurcation diagrams can be obtained by varying different system parameters, and the system exhibits not only a typical period-doubling route to chaos but also the period-3 window. Analytical modeling of period-1 and hence period- $p$ orbits as well as their stability analysis using the characteristic multipliers are presented. Thus, those stable ranges of various system parameters can be formulated, and hence the chaotic ranges can be determined. The theoretical results are verified by using both PSpice simulation and experimental measurement.
\end{abstract}

\section{Introduction}

One basic belief in our common sense is that simple systems exhibit simple behavior. In fact, investigation of some simple switching dc-dc buck converters has revealed remarkable complex behavior, namely chaos [1]-[5].

The investigation onto chaotic behavior in power electronics circuits was launched in late 1980's. Since linear system theory is ill-suited to investigate the subharmonics and chaotic phenomena occurred in power electronics circuits, the most attractive approach has been the iterative nonlinear mapping. The one-dimensional mapping was successfully employed to derive the chaotic region of switching dc-dc converters in which the load was assumed as constant-voltage sink [1]-[3]. However, this load voltage is usually non-constant for practical switching dc-dc converters, leading to be a second-order rather than a first-order system.

In order to investigate the chaotic behavior of practically switching dc-dc converters, the corresponding twodimensional mapping has recently been manipulated [4],[5]. However, most of them have been focused on the derivation of the period-1 and period-2 orbits only. Even so, the corresponding characteristic multipliers need to be numerically computed.

On the other hand, the investigation onto chaotic behavior of industrial motor drives has been surprisingly little [6],[7]. Nevertheless, the chaotic behavior of PWM inverter-fed induction motor drives has recently been investigated by using numerical analysis [6]. It has also been attempted to investigate the chaotic behavior of brushless dc motor drives by ignoring the switching effect and approximately transforming into the Lorenz system [7]. To the best of the authors' knowledge, analytical investigation onto industrial motor drives is absent in power electronics literature. The major reason should be due to the complexity of analytical formulation when considering the nonlinearity caused by the switching effect.
It is the purpose of this paper to investigate, both numerically and analytically, the nonlinear dynamics and chaotic behavior of industrial motor drives without ignoring the switching effect or accepting rough assumptions. A simple dc chopper-fed permanent-magnet (PM) dc motor drive is targeted for investigation which forms the basis for investigating other industrial motor drives.

Firstly, a generalized two-dimensional iterative map that describes the nonlinear dynamics of a second-order dc drive system operating in the continuous condition mode will be derived. This derivation will cover all possible solutions, such as real and complex roots, to handle different system parameters and conditions. Secondly, analytical modeling of the period- 1 and hence the generalized period- $p$ orbits as well as their stability analysis will be presented. Thirdly, based on the derived iterative map, computer simulations will be carried out to study the chaotic behavior and possible route to chaos. Finally, a practical drive system will be PSpice-simulated and experimentally tested for verification.

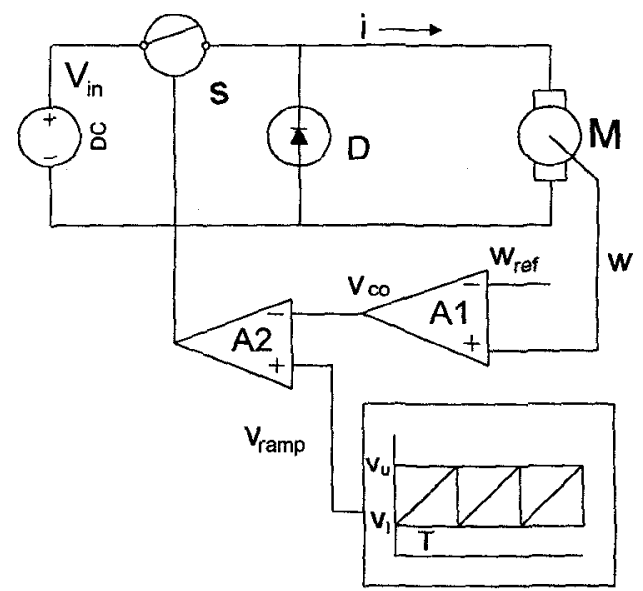

Fig. 1: Block diagram of dc drive

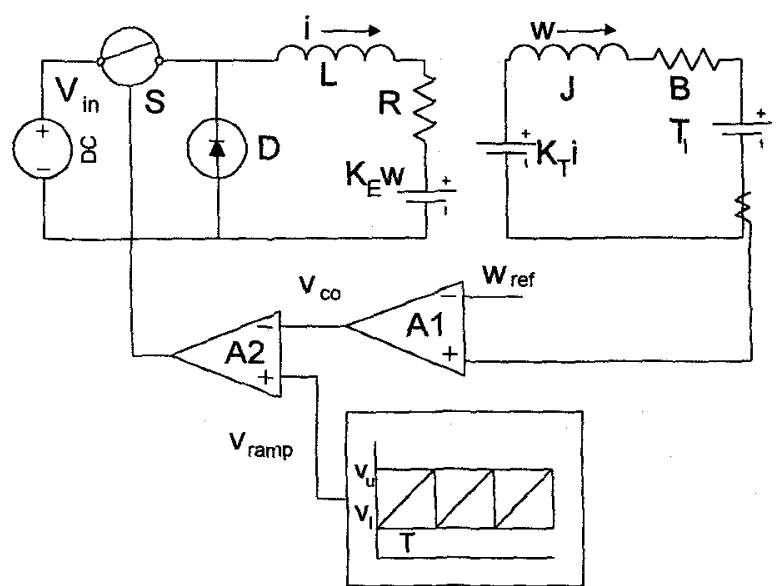

Fig. 2: Equivalent circuit of dc drive 


\section{Modeling of Drive Systems}

As shown in Fig. 1, a simple buck-type dc chopper-fed PM $\mathrm{dc}$ motor drive operating in the continuous condition mode is used for exemplification. The corresponding equivalent circuit is shown in Fig. 2 which will be used throughout the analysis. The rotor speed $\omega$ of the PM dc motor is controlled by naturally-sampled constant-frequency pulse-width modulation (PWM).

Consider that the operational amplifier $\mathrm{A}_{1}$ has a feedback gain $g$, the control signal $v_{c o}$ can be expressed as

$v_{c o}(t)=g\left(\omega(t)-\omega_{\text {ref }}\right)$

where $\omega(t)$ and $\omega_{\text {ref }}$ are the instantaneous and reference speeds of the motor, respectively. The ramp voltage $v_{\text {ramp }}$ is represented by

$v_{\text {ramp }}(t)=v_{l}+\left(v_{u}-v_{l}\right) t / T$

where $v_{l}$ and $v_{u}$ are respectively the lower and upper voltages of the ramp signal, and $T$ is its period. Then, both $v_{c o}$ and $v_{\text {ramp }}$ are fed into the comparator $\mathrm{A}_{2}$ which outputs the signal to turn on or off the power switch $S$. When the control voltage exceeds the ramp voltage, $S$ is off and the diode $D$ is on; otherwise, $S$ is on and $D$ is off. Thus, the system equation can be divided into two stages as given by

- Stage 1: $v_{c o} \geq v_{\text {ramp }}$,

$$
\frac{d}{d t}\left(\begin{array}{c}
\omega(t) \\
i(t)
\end{array}\right)=\left(\begin{array}{cc}
-B / J & K_{T} / J \\
-K_{E} / L & -R / L
\end{array}\right)\left(\begin{array}{c}
\omega(t) \\
i(t)
\end{array}\right)+\left(\begin{array}{c}
-T_{l} / J \\
0
\end{array}\right)
$$

- Stage 2: $v_{c o} \leq v_{\text {ramp }}$,

$$
\frac{d}{d t}\left(\begin{array}{c}
\omega(t) \\
i(t)
\end{array}\right)=\left(\begin{array}{cc}
-B / J & K_{T} / J \\
-K_{E} / L & -R / L
\end{array}\right)\left(\begin{array}{c}
\omega(t) \\
i(t)
\end{array}\right)+\left(\begin{array}{c}
-T_{l} / J \\
V_{i n} / L
\end{array}\right)
$$

where $i$ is armature current, $R$ armature resistance, $L$ armature inductance, $V_{\text {in }}$ dc supply voltage, $K_{E}$ back-EMF constant, $K_{T}$ torque constant, $B$ viscous damping, $J$ load inertia, and $T_{l}$ load torque.

By defining the state vector $\mathbf{X}(t)$ and the following matrices $\mathbf{A}, \mathbf{E}_{1}, \mathbf{E}_{2}$

$$
\begin{aligned}
& \mathbf{A}=\left(\begin{array}{cc}
-B / J & K_{T} / J \\
-K_{E} / L & -R / L
\end{array}\right), \mathbf{X}(t)=\left(\begin{array}{c}
\omega(t) \\
i(t)
\end{array}\right) \\
& \mathbf{E}_{1}=\left(\begin{array}{c}
-T_{l} / J \\
0
\end{array}\right), \mathbf{E}_{2}=\left(\begin{array}{c}
-T_{l} / J \\
V_{\text {in }} / L
\end{array}\right)
\end{aligned}
$$

the system equation can be rewritten as

$$
\dot{\mathbf{X}}(t)=\mathbf{A} \mathbf{X}(t)+\mathbf{E}_{k} \quad(k=1,2)
$$

As $k$ changes the value when $v_{c o}=v_{\text {ramp }}$ while $v_{c o}$ is time dependent, the system equation given by (7) is in fact a timevarying state equation. Thus, the closed-loop drive system is a second-order non-autonomous dynamical system.

\section{Analysis of Chaotic Behavior}

Since the drive system operates periodically with $T, \mathbf{X}(t)$ is generally sampled at the beginning of every ramp cycle. Thus, the corresponding discrete state vector is denoted as $\mathbf{X}_{n}=\mathbf{X}(n T)$. The iterative function that maps this vector to its successive one $\mathbf{X}_{n+1}=\mathbf{X}((n+1) T)$ is called the Poincaré map which is defined as $P: \mathfrak{R}^{2} \rightarrow \mathfrak{R}^{2}$

$\mathbf{X}_{n+1}=P\left(\mathbf{X}_{n}\right)$

\subsection{Analytical solution of system equation}

Given the desired initial conditions $\mathbf{X}\left(t_{0}\right)$, the analytical solution of the system equation given by (7) can be expressed as

$$
\begin{aligned}
\mathbf{X}(t) & =\Phi\left(t-t_{0}\right) \mathbf{X}\left(t_{0}\right)+\int_{t_{0}}^{t} \Phi(t-\tau) \mathbf{E}_{k} d \tau \\
& =-\mathbf{A}^{-1} \mathbf{E}_{k}+\Phi\left(t-t_{0}\right)\left(\mathbf{X}\left(t_{0}\right)+\mathbf{A}^{-1} \mathbf{E}_{k}\right) \quad(k=1,2)
\end{aligned}
$$

where $\Phi(t)=e^{\mathrm{A} t}$ is so-called the state transition matrix.

By defining the following parameters

$$
\alpha=\frac{1}{2}\left(\frac{R}{L}+\frac{B}{J}\right), \Delta=\frac{1}{4}\left(\frac{R}{L}-\frac{B}{J}\right)^{2}-\frac{K_{E} K_{T}}{L J}
$$

the eigenvalues $\lambda_{1}, \lambda_{2}$ of the matrix $\mathbf{A}$ can be expressed as

for $\Delta=0, \lambda_{1}=\lambda_{2}=\lambda=-\alpha$

for $\Delta>0, \lambda_{1}=-\alpha+\sqrt{\Delta}, \lambda_{2}=-\alpha-\sqrt{\Delta}$

for $\Delta<0, \lambda_{1}=-\alpha+j \sqrt{-\Delta}, \lambda_{2}=-\alpha-j \sqrt{-\Delta}$

Hence, the corresponding state transition matrix can be obtained as

for $\Delta=0, \Phi(t)=e^{-\alpha}[1-t(2 \alpha 1-\mathbf{A})]$

for $\Delta>0, \Phi(t)=\frac{1}{\lambda_{2}-\lambda_{1}}\left[e^{\lambda_{1} t}\left(\lambda_{2} \mathbf{1}-\mathbf{A}\right)-e^{\lambda_{2} t}\left(\lambda_{1} \mathbf{1}-\mathbf{A}\right)\right]$

for $\Delta<0, \Phi(t)=e^{-\alpha t}\left[1 \cos (\beta t)+\frac{1}{\beta}(\alpha 1+\mathbf{A}) \sin (\beta t)\right]$

where 1 is the identity matrix and $\beta=\sqrt{-\Delta}$.

\subsection{Derivation of the generalized Poincare map}

Within each $T$, there are two possible situations - a skipped cycle because of the absence of intersection between $v_{c o}$ and $v_{\text {ramp; }}$; and an intersected cycle in which there is at least one intersection between $v_{c o}$ and $v_{\text {ramp }}$.

For the skipped cycle, $\mathrm{S}$ does not change its state, remaining either on or off. The corresponding Poincaré map $P$ can be easily derived from (9) as given by

$\mathbf{X}_{n+1}=-\mathbf{A}^{-1} \mathbf{E}_{k}+\Phi(T)\left(\mathbf{X}_{n}+\mathbf{A}^{-1} \mathbf{E}_{k}\right) \quad(k=1,2)$

For the intersected cycle, $\mathrm{S}$ may change $m$ times when $v_{c o}$ crosses $v_{\text {ramp }}$ by $m$ times within the same $T$, so-called multiple pulsing. The corresponding intersections occur at $t=n T+\delta_{i} T, \quad 0=\delta_{0}<\delta_{1}<, \cdots,<\delta_{m}<1 \quad(i=1, \cdots, m)$

Hence, the generalized Poincaré map can be expressed as the following iterative form

$\mathbf{X}\left(n T+\delta_{i} T\right)=\mathbf{f}_{i}\left(\mathbf{X}\left(n T+\delta_{i-1} T\right), \delta_{i-1} T, \delta_{i} T\right)(i=1, \cdots, m)$

$\mathbf{X}_{n+1}=\mathbf{f}_{m+1}\left(\mathbf{X}\left(n T+\delta_{m} T\right), \delta_{m} T, T\right)$

For $m=1$, if $v_{c o}\left(t_{n}\right)>v_{l}$, the drive system operates in Stage 1 from $n T$ to $n T+\delta_{1} T$, at which the transition condition $\left(v_{c o}=v_{\text {ramp }}\right)$ is satisfied, and then in Stage 2 till $(n+1) T$. Using (1), (2) and (9), $\delta_{1}$ can be determined by evaluating the solution of the following transcendental equation

$$
\begin{aligned}
h\left(\delta_{1}\right)= & \omega_{r e f}+\left(v_{l}+\left(v_{u}-v_{l}\right) \delta_{1}\right) / g \\
& -(1,0)\left[-\mathbf{A}^{-1} \mathbf{E}_{1}+\Phi\left(\delta_{1} T\right)\left(\mathbf{X}_{n}+\mathbf{A}^{-1} \mathbf{E}_{1}\right)\right]=0
\end{aligned}
$$

Hence, the corresponding Poincaré map can be written as

$\mathbf{X}\left(n T+\delta_{1} T\right)=-\mathbf{A}^{-1} \mathbf{E}_{1}+\Phi\left(\delta_{1} T\right)\left(\mathbf{X}_{n}+\mathbf{A}^{-1} \mathbf{E}_{1}\right)$

$\mathbf{X}_{n+1}=-\mathbf{A}^{-1} \mathbf{E}_{2}+\Phi\left(T-\delta_{1} T\right)\left(\mathbf{X}\left(n T+\delta_{1} T\right)+\mathbf{A}^{-1} \mathbf{E}_{2}\right)$

On the contrary, if $v_{c o}\left(t_{n}\right)<v_{l}$, the drive system operates firstly in Stage 2 and then switches to Stage 1. The corresponding Poincaré map can similarly be obtained as 


$$
\begin{aligned}
h\left(\delta_{1}\right)= & \omega_{r e f}+\left(v_{l}+\left(v_{u}-v_{l}\right) \delta_{1}\right) / g \\
& -(1,0)\left[-\mathbf{A}^{-1} \mathbf{E}_{2}+\Phi\left(\delta_{1} T\right)\left(\mathbf{X}_{n}+\mathbf{A}^{-1} \mathbf{E}_{2}\right)\right]=0 \\
\mathbf{X}(n T+ & \left.\delta_{1} T\right)=-\mathbf{A}^{-1} \mathbf{E}_{2}+\Phi\left(\delta_{1} T\right)\left(\mathbf{X}_{n}+\mathbf{A}^{-1} \mathbf{E}_{2}\right) \\
\mathbf{X}_{n+1}= & -\mathbf{A}^{-1} \mathbf{E}_{1}+\Phi\left(T-\delta_{1} T\right)\left(\mathbf{X}\left(n T+\delta_{1} T\right)+\mathbf{A}^{-1} \mathbf{E}_{1}\right)
\end{aligned}
$$

Similarly to the derivation for $m=1$, the generalized Poincaré map for $m>1$ can be obtained as

$$
\begin{aligned}
h\left(\delta_{i}\right)= & \omega_{r e f}+\left(v_{i}+\left(v_{u}-v_{l}\right) \delta_{i}\right) / g-(1,0)\left[-\mathbf{A}^{-1} \mathbf{E}_{k}\right. \\
& \left.+\Phi\left(\delta_{i} T-\delta_{i-1} T\right)\left(\mathbf{X}\left(n T+\delta_{i-1} T\right)+\mathbf{A}^{-1} \mathbf{E}_{k}\right)\right]=0
\end{aligned}
$$

$\mathbf{X}\left(n T+\delta_{i} T\right)=-\mathbf{A}^{-1} \mathbf{E}_{k}+\Phi\left(\delta_{i} T-\delta_{i-1} T\right)\left(\mathbf{X}\left(n T+\delta_{i-1} T\right)+\mathbf{A}^{-1} \mathbf{E}_{k}\right)$

$\mathbf{X}_{n+1}=-\mathbf{A}^{-1} \mathbf{E}_{k}+\Phi\left(T-\delta_{m} T\right)\left(\mathbf{X}\left(n T+\delta_{m} T\right)+\mathbf{A}^{-1} \mathbf{E}_{k}\right)$

where $i=1, \cdots, m$, and $k$ equals 1 or 2 depending on whether $v_{c o}$ is over $v_{\text {ramp }}$ or not.

It should be noted that the above generalized Poincare map is derived from the analytical solution of the model of dc drive systems which covers all possible solutions such as real and complex roots due to different system parameters and conditions. Thus, this Poincaré map can be considered as the map for any second-order dynamical systems using similar mathematical models, such as other switching power converters and dc motor drives. Moreover, the derivation can readily be extended to those higher-order dynamical systems involving power switches.

\subsection{Analysis of periodic orbits}

The above generalized Poincaré map is so general that it includes cycle skipping and multiple pulsing. Due to their presence, it is very inconvenient to analyze the steady-state periodic orbits and their stability. Also, the presence of multiple pulsing can greatly increase the switching losses, which should be avoided by using a latch or sample-and-hold. Therefore, instead of using the generalized one, the detailed analysis of periodic orbits will adopt a specific Poincaré map that the orbits cross the ramp signal once per cycle mathematically $m=1$.

The steady-state periodic solution of the dc drive system can be a fixed point, so-called the period-1 orbit $\mathbf{X}^{*}$, or a cycle point, so-called the period- $p$ orbit $\left\{\mathbf{X}_{1}^{*}, \cdots, \mathbf{X}_{p}^{*}\right\} \quad(p>1)$. The corresponding specific Poincaré maps are described as $\mathbf{X}^{*}=P\left(\mathbf{X}^{*}\right)$

$\mathbf{X}_{k+1}^{*}=P\left(\mathbf{X}_{k}^{*}\right) \quad(k=1, \cdots, p-1), \quad \mathbf{X}_{1}^{*}=P\left(\mathbf{X}_{p}^{*}\right)$

Firstly, the period- 1 orbit is analyzed. Since $m=1$ and the orbit must start with $v_{l}<v_{c o}<v_{u}$, the corresponding Poincare map can be deduced from (22) and (23) as given by

$$
\begin{aligned}
\mathbf{X}_{n+1}= & -\mathbf{A}^{-1} \mathbf{E}_{1}+\Phi(T)\left(\mathbf{X}_{n}+\mathbf{A}^{-1} \mathbf{E}_{1}\right) \\
& -\left(\mathbf{1}-\Phi\left(T-\delta_{1} T\right)\right) \mathbf{A}^{-1}\left(\mathbf{E}_{2}-\mathbf{E}_{1}\right)
\end{aligned}
$$

Substituting (32) into the map given by (30), the period-1 orbit can be obtained as

$$
\mathbf{X}^{*}=-\mathbf{A}^{-1} \mathbf{E}_{1}-(\mathbf{1}-\Phi(T))^{-1}\left(\mathbf{1}-\Phi\left(T-\delta_{1} T\right)\right) \mathbf{A}^{-1}\left(\mathbf{E}_{2}-\mathbf{E}_{1}\right)
$$

After substitution of (33) into (21), $\delta_{1}$ can be determined from the corresponding transcendental equation

$$
\begin{gathered}
h\left(\delta_{1}\right)=\omega_{r e f}+\left(v_{1}+\left(v_{u}-v_{l}\right) \delta_{1}\right) / g-(1,0)\left[-\mathbf{A}^{-1} \mathbf{E}_{1}-\Phi\left(\delta_{1} T\right)\right. \\
\\
\left.(\mathbf{1}-\Phi(T))^{-1}\left(\mathbf{1}-\Phi\left(T-\delta_{1} T\right)\right) \mathbf{A}^{-1}\left(\mathbf{E}_{2}-\mathbf{E}_{1}\right)\right]=0
\end{gathered}
$$

Hence, provided that $\delta_{1} \in(0,1), \mathbf{X}^{*}$ can be obtained from (33). Notice that $\mathbf{X}^{*}$ is the period- 1 orbit if and only if $v_{c o}$ always lies between $v_{l}$ and $v_{u}$.

Secondly, the period- $p$ orbit $\left\{\mathbf{X}_{1}^{*}, \cdots, \mathbf{X}_{p}^{*}\right\}$ is analyzed. By defining $\delta_{1}, \cdots, \delta_{p}$ as the duty cycles of $p$ periods with $p>1$, it indicates that $v_{c o}(t)$ crosses the voltage ramp $p$ times at $\left(n T+\delta_{1} T\right), \cdots,\left((n+p) T+\delta_{p} T\right)$, respectively. Based on the specific Poincaré map derived in (32), the $p$-fold iterative Poincaré map can be formulated as

$$
\begin{aligned}
\mathbf{X}_{n+p}= & p^{(p)}\left(\mathbf{X}_{n}\right) \\
= & -\mathbf{A}^{-1} \mathbf{E}_{1}+\Phi(p T)\left(\mathbf{X}_{n}+\mathbf{A}^{-1} \mathbf{E}_{1}\right) \\
& -\sum_{j=1}^{p}\left[\Phi((p-j) T)\left(\mathbf{1}-\Phi\left(T-\delta_{j} T\right)\right] \mathbf{A}^{-1}\left(\mathbf{E}_{2}-\mathbf{E}_{1}\right)\right.
\end{aligned}
$$

By using (31), the period- $p$ orbit can then be derived as

$$
\begin{aligned}
\mathbf{X}_{1}^{*}= & -\mathbf{A}^{-1} \mathbf{E}_{1}-(\mathbf{1}-\Phi(p T))^{-1} \sum_{j=1}^{p}[\Phi((p-j) T) \\
& \left(\mathbf{1}-\Phi\left(T-\delta_{j} T\right)\right] \mathbf{A}^{-1}\left(\mathbf{E}_{2}-\mathbf{E}_{1}\right) \\
\mathbf{X}_{i}^{*}=- & \mathbf{A}^{-1} \mathbf{E}_{1}+\Phi((i-1) T)\left(\mathbf{X}_{1}^{*}+\mathbf{A}^{-1} \mathbf{E}_{1}\right)-\sum_{j=1}^{i-1}[\Phi((i-j-1) T) \\
& \left(\mathbf{1}-\Phi\left(T-\delta_{j} T\right)\right] \mathbf{A}^{-1}\left(\mathbf{E}_{2}-\mathbf{E}_{\mathfrak{i}}\right) \quad(i=2, \cdots, p) \\
h_{i}(\mathbf{d})= & \omega_{r e f}+\left(v_{l}+\left(v_{u}-v_{l}\right) \delta_{i}\right) / g-(1,0)\left[-\mathbf{A}^{-1} \mathbf{E}_{1}\right. \\
& \left.+\Phi\left(\delta_{i} T\right)\left(\mathbf{X}_{i}^{*}+\mathbf{A}^{-1} \mathbf{E}_{1}\right)\right]=0 \quad(i=1, \cdots, p)
\end{aligned}
$$

where $\Phi(0)=1$ and $\mathbf{d}=\left(\delta_{1}, \cdots, \delta_{p}\right)$. By substituting (36) and (37) into (38), $\mathbf{d}$ can be determined. Provided that $\delta_{i} \in(0,1)$ for $i=1, \cdots, m, \mathbf{X}_{1}^{*}, \cdots, \mathbf{X}_{p}^{*}$ can then be obtained from (36) and (37). If the condition $v_{l}<v_{c o}<v_{u}$ is guaranteed in each period, $\left\{\mathbf{X}_{1}^{*}, \cdots, \mathbf{X}_{p}^{*}\right\}$ is a period-p orbit.

Due to the cyclic property of a cycle point, $\left\{\mathbf{X}_{2}^{*}, \cdots, \mathbf{X}_{p}^{*}, \mathbf{X}_{1}^{*}\right\}, \cdots,\left\{\mathbf{X}_{p}^{*}, \mathbf{X}_{1}^{*}, \cdots, \mathbf{X}_{p-1}^{*}\right\}$ are also period- $p$ orbits which correspond to the same subharmonic frequency. If $\mathbf{X}_{\mathrm{I}}^{*}=\cdots=\mathbf{X}_{p}^{*}$, the period- $p$ orbit becomes the period-1 orbit, which shows that period-1 is its subset.

\subsection{Stability and characteristic multipliers}

For both the period- 1 and period- $p$ orbits, they may be stable or unstable. It is known that the stability type of a fixed point of mapping corresponds to the stability type of the underlying periodic solution, and the fixed point of mapping is stable if and only if its characteristic multipliers all lie within the unit circle in the complex plane.

For the period-1 orbit that is a fixed point of the specific Poincaré map given by (32), its characteristic multipliers are the eigenvalues of the Jacobian matrix of that mapping, which is given by

$$
\begin{aligned}
D P\left(\mathbf{X}^{*}\right) & =\Phi(T)+\frac{\partial \Phi\left(T-\delta_{1} T\right)}{\partial \delta_{1}} \mathbf{A}^{-1}\left(\mathbf{E}_{2}-\mathbf{E}_{1}\right) \frac{\partial \delta_{1}}{\partial \mathbf{X}^{*}} \\
& =\Phi(T)-\Phi\left(T-\delta_{1} T\right)\left(\mathbf{E}_{2}-\mathbf{E}_{1}\right) T \frac{\partial \delta_{1}}{\partial \mathbf{X}^{*}}
\end{aligned}
$$

where $\frac{\partial \delta_{1}}{\partial \mathbf{X}^{*}}=\left(\frac{\partial \delta_{1}}{\partial \omega}, \frac{\partial \delta_{1}}{\partial i}\right)$. According to the implicit-function theorems, $\frac{\partial \delta_{1}}{\partial \mathbf{X}^{*}}$ can be deduced from (21) as 


$$
\begin{aligned}
\frac{\partial \delta_{1}}{\partial \mathbf{X}^{*}} & =-\left(\frac{\partial h}{\partial \delta_{1}}\right)^{-1} \frac{\partial h}{\partial \mathbf{X}^{*}} \\
& =\left[\left(v_{u}-v_{l}\right) / g-(1,0)\left(\Phi\left(\delta_{1} T\right)\left(\mathbf{A} \mathbf{X}^{*}+\mathbf{E}_{1}\right) T\right]^{-1}(1,0) \Phi\left(\delta_{1} T\right)\right.
\end{aligned}
$$

For the period- $p$ orbit $\left\{\mathbf{X}_{1}^{*}, \cdots, \mathbf{X}_{p}^{*}\right\}, \mathbf{X}_{1}^{*}$ is a fixed point of the $p$-fold iterative specific Poincaré map. Therefore, its characteristic multipliers are the eigenvalues of the Jacobian matrix of that mapping, which is given by

$$
\begin{aligned}
& D P^{(p)}\left(\mathbf{X}_{1}^{*}\right) \\
& \quad=\Phi(p T)-\sum_{j=1}^{p} \Phi((p-j) T) \Phi\left(T-\delta_{j} T\right)\left(\mathbf{E}_{2}-\mathbf{E}_{1}\right) T \frac{\partial \delta_{j}}{\partial \mathbf{X}_{1}^{*}}
\end{aligned}
$$

By substituting (37) into (38), it results

$\mathbf{H}\left(\mathbf{d}, \mathbf{X}_{1}^{*}\right)=\left[h_{1}\left(\mathbf{d}, \mathbf{X}_{1}^{*}\right), \cdots, h_{p}\left(\mathbf{d}, \mathbf{X}_{1}^{*}\right)\right]^{T}=\mathbf{0}$

Hence, according to the implicit-function theorems, the partial derivative in (41) can be expressed as

$$
\frac{\partial \mathrm{d}}{\partial \mathbf{X}_{1}^{*}}=\left(\frac{\partial \delta_{1}}{\partial \mathbf{X}_{1}^{*}}, \cdots, \frac{\partial \delta_{p}}{\partial \mathbf{X}_{1}^{*}}\right)^{T}=-\left[\frac{\partial \mathbf{H}}{\partial \mathbf{d}}\right]^{-1}\left[\frac{\partial \mathbf{H}}{\partial \mathbf{X}_{1}^{*}}\right]
$$

where

$$
\begin{aligned}
\frac{\partial \mathbf{H}}{\partial \mathbf{d}}= & {\left[\frac{\partial h_{i}}{\partial \delta_{j}}\right]_{p \times p} } \\
= & {\left[\left(v_{u}-v_{l}\right) / g\right] \mathbf{1}-\operatorname{diag}\left[(1,0) \Phi\left(\delta_{i} T\right)\left(\mathbf{A} \mathbf{X}_{i}^{*}+\mathbf{E}_{1}\right) T\right] } \\
& +\left[(1,0) \Phi\left(\delta_{i} T\right) \Phi\left((i-j) T-\delta_{j} T\right)\left(\mathbf{E}_{2}-\mathbf{E}_{1}\right) T\right]_{p \times p} \\
\frac{\partial \mathbf{H}}{\partial \mathbf{X}_{1}^{*}}= & {\left[\frac{\partial h_{1}}{\partial \mathbf{X}_{1}^{*}}, \cdots, \frac{\partial h_{p}}{\partial \mathbf{X}_{1}^{*}}\right]^{T} } \\
= & -\left[(1,0) \Phi\left(\delta_{1} T\right), \cdots,(1,0) \Phi\left((p-1) T+\delta_{p} T\right)\right]^{T}
\end{aligned}
$$

Notice that $\Phi\left((i-j) T-\delta_{j} T^{\prime}\right)$ in (44) becomes a zero matrix when $j \geq i$.

\section{Computer Simulations}

To illustrate the derived iterative maps, computer simulations are carried out. The simulation parameters are based on the values of a practical dc chopper-fed PM dc motor drive system. For this set of practical parameters, the eigenvalues of matrix $\mathbf{A}$ are $\lambda_{1}=-6.35$ and $\lambda_{2}=-90.24$. These eigenvalues indicate that the mechanical speed of the drive system will response much slower than the electrical current because the mechanical time constant $\left(1 / \lambda_{1}\right)$ is far greater than the electrical time constant $\left(1 / \lambda_{2}\right)$.

\subsection{Bifurcation diagrams using numerical computation}

By employing standard numerical techniques such as the brute-force algorithm [8] to compute the generalized Poincaré map given by (27)-(29), bifurcation diagrams of the speed versus input voltage, the speed versus gain and the armature current versus gain can be resulted as shown in Figs. 3, 4 and 5 , respectively.

As reflected from these figures, the system exhibits a typical period-doubling route to chaos, covering both chaotic speed and current. Since the motor speed is dictated by the armature current, it can be found that the speed bifurcation with respect to gain shown in Fig. 4 is similar in shape to the current bifurcation with respect to gain in Fig. 5. Moreover, because of the great difference between the mechanical and electrical time constants, the actual fluctuation of the motor speed shown in Fig. 4 (about 100rad/s to $101.75 \mathrm{rad} / \mathrm{s}$ ) which is far smaller than that of the armature current shown in Fig. 5 (about $1 \mathrm{~A}$ to $7 \mathrm{~A}$ ). It is interesting to note that the bifurcation diagrams shown in Figs. 4 and 5 exhibit the period-3 and period -6 orbits even after reaching chaos.

The strange attractor of the armature current versus motor speed with $g=3.4$ is also displayed in Fig. 6 .

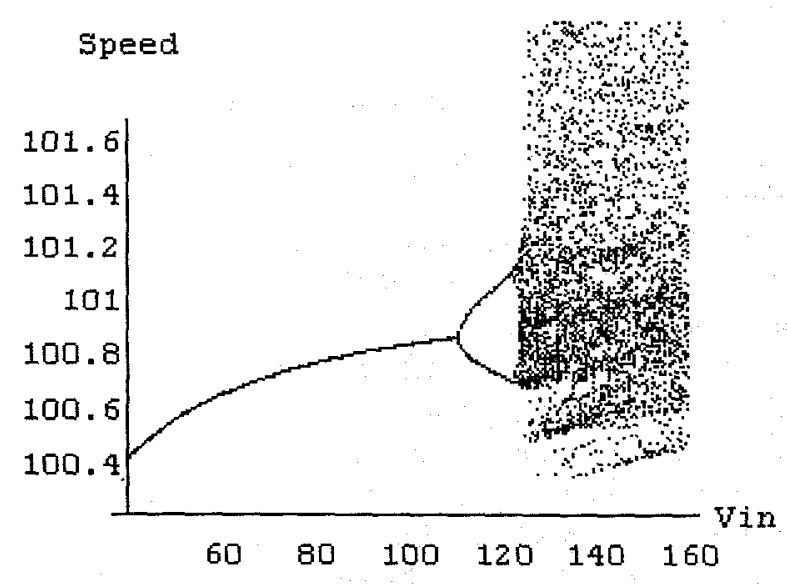

Fig. 3: Bifurcation diagram motor speed versus input voltage

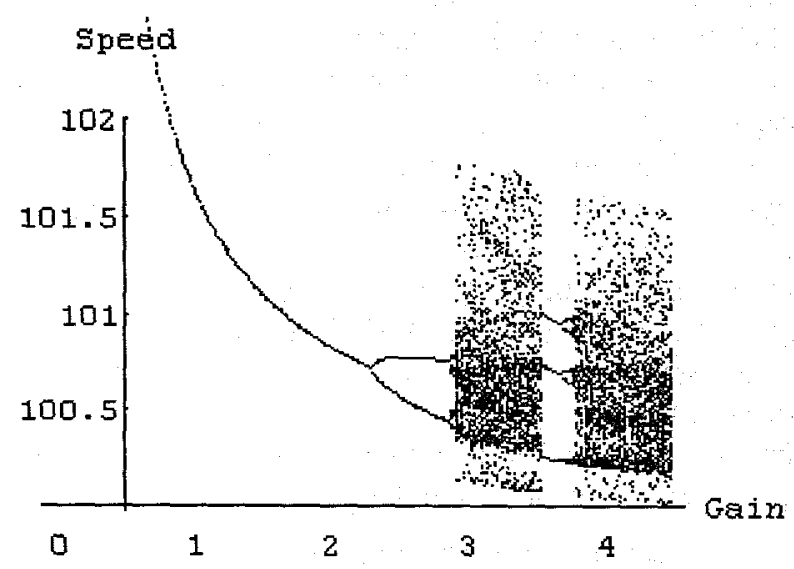

Fig. 4: Bifurcation diagram of motor speed versus gain

Current

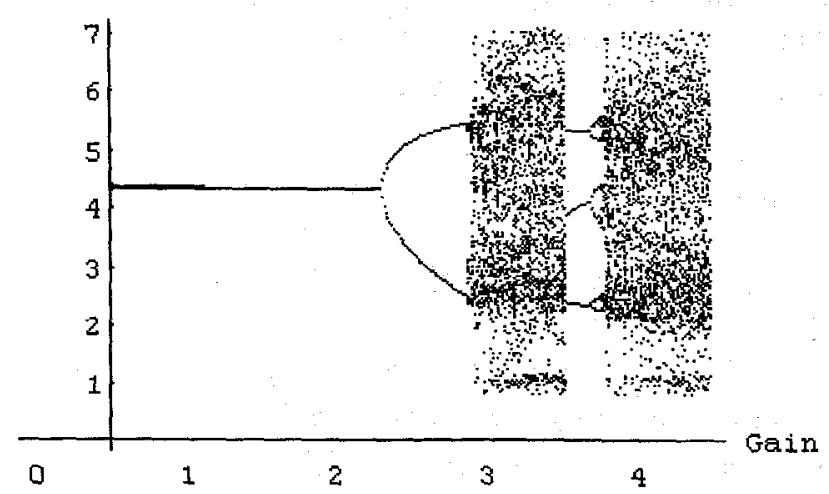

Fig. 5: Bifurcation diagram of armature current versus gain 


\subsection{Periodic and chaotic waveforms using PSpice}

Based on the same equivalent circuit shown in Fig. 2, realistic time-domain waveforms can be obtained by using PSpice simulation. As shown in Fig. 7, the PSpice-simulated waveforms of $v_{c o}$ and $v_{\text {ramp }}$ at $g=1.5$ illustrate that the system is operating at the period-1 orbit. When $g=3.4$, the waveform of $i$ shown in Fig. 7 is in chaos. These system behaviors closely agree with theoretical bifurcation diagrams in Figs. 4 and 5.

Current

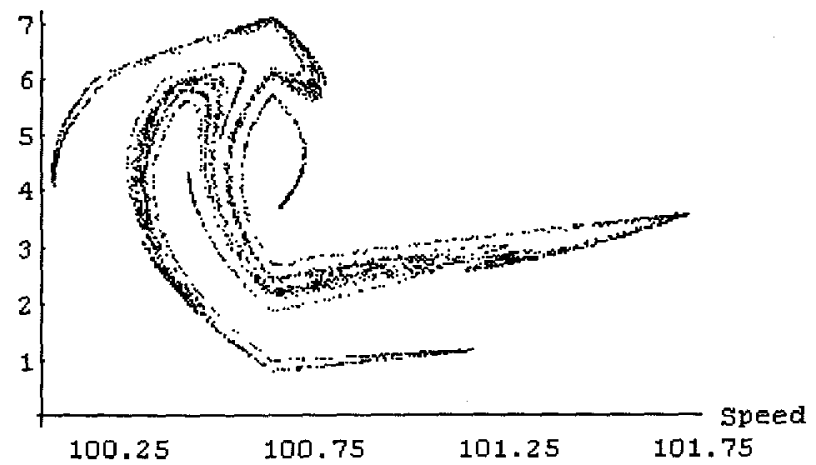

Fig. 6: Strange attractor of armature current and motor speed

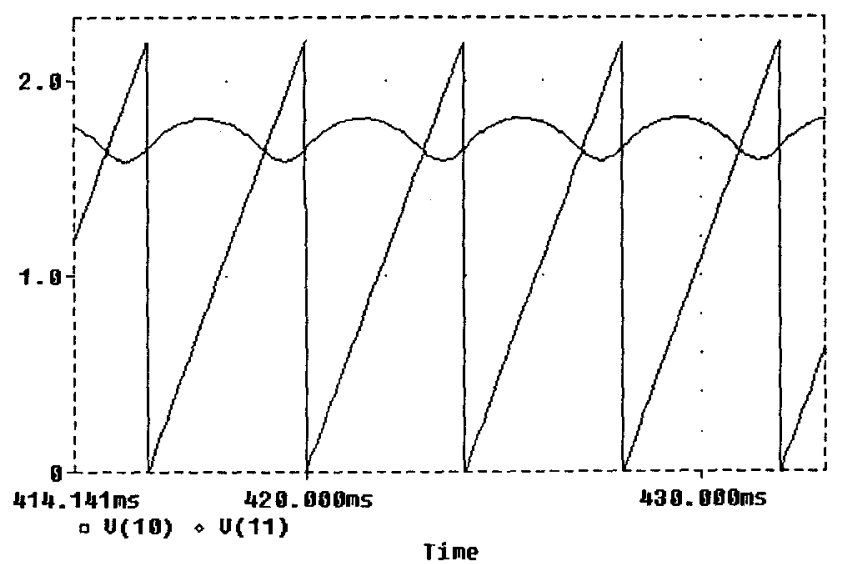

Fig. 7: PSpice-simulated waveforms of control voltage and ramp voltage during period-1 operation

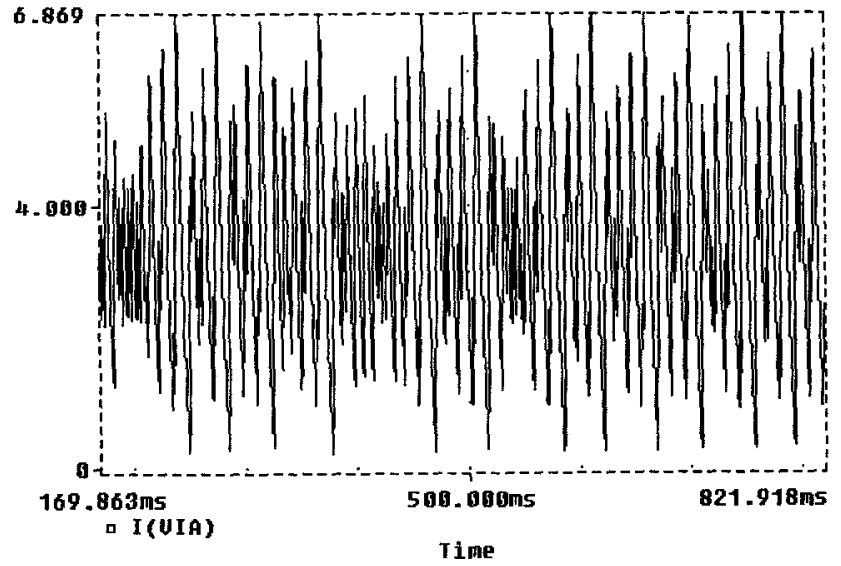

Fig. 8: PSpice-simulated waveform of armature current during chaotic operation

\subsection{Bifurcation diagrams using analytical approach}

Based on the derived relationship in (34) for the period-1 orbit, the solution of $\delta_{1}$ for a range of $V_{\text {in }}$ varying from $40 \mathrm{~V}$ to $160 \mathrm{~V}$ is shown in Fig. 9. Hence, by using (39) and (40), the corresponding eigenvalues of the Jacobian matrix $D P$ is shown in Fig. 10 in which one of the amplitudes is greater than 1 when $V_{i n}>110.8 \mathrm{~V}$. It indicates that the period-1 orbit is unstable when $V_{\text {in }}>110.8 \mathrm{~V}$.

Similarly, based on (38) with $p=2$, the solution of $\left\{\delta_{1}, \delta_{2}\right\}$ for a range of $V_{\text {in }}$ from $100 \mathrm{~V}$ to $160 \mathrm{~V}$ is shown in Fig. 11. It can be found that the period-2 orbit occurs when $V_{i n}>110.8 \mathrm{~V}$ and one of the duty cycles is greater than 1 after $V_{i n}>122.8 \mathrm{~V}$. Thus, the period-2 orbit only exists between $110.8 \mathrm{~V}$ and $122.8 \mathrm{~V}$. Moreover, by using (41)-(45) with $p=2$, the corresponding eigenvalues for the period-2 orbit are shown in Fig. 12. It indicates that this period-2 orbit lying between $110.8 \mathrm{~V}$ and $122.8 \mathrm{~V}$ is stable.

It should be noted that the above analytical results closely agree with the bifurcation diagram (Fig. 3) resulting from numerical computation. The required computational time based on the derived analytical solution is extremely less than that required for computation using the brute-force algorithm. Therefore, it greatly facilitates the identification of the desired stable operating ranges for different system parameters and conditions.

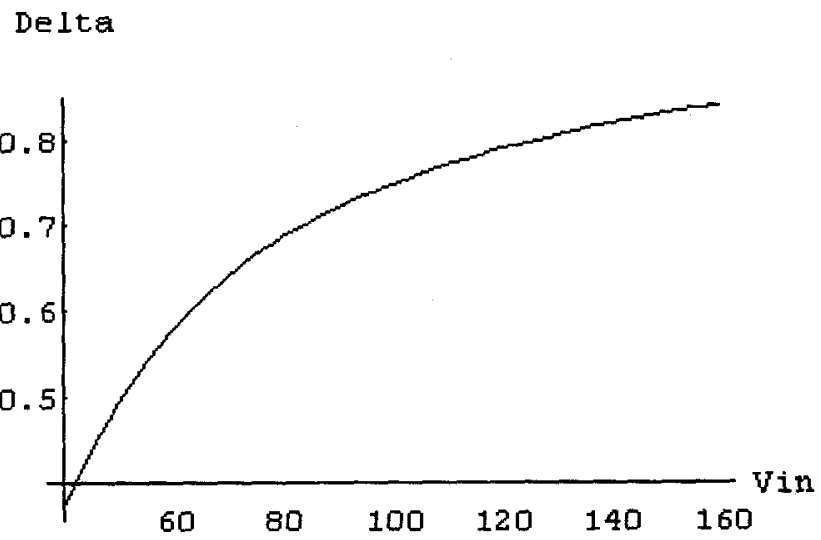

Fig. 9: Evolution of duty cycle for the period-one orbit

\section{Eigenvalues}

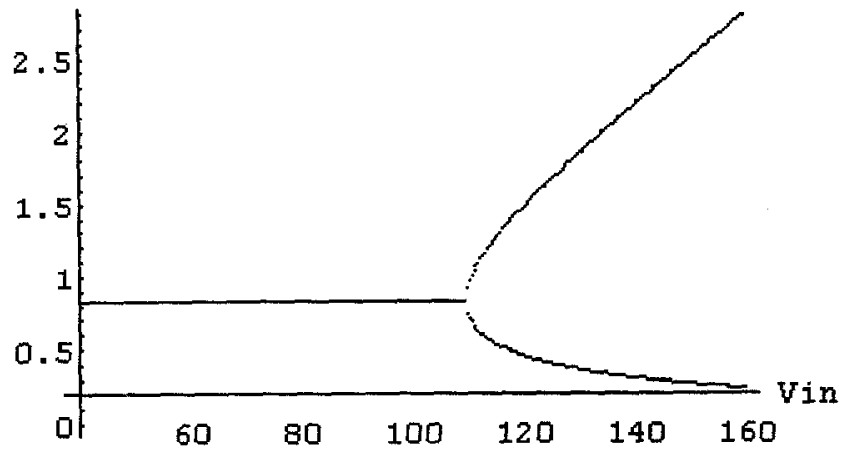

Fig. 10: Evolution of characteristic multipliers for the period-2 orbit 
Delta

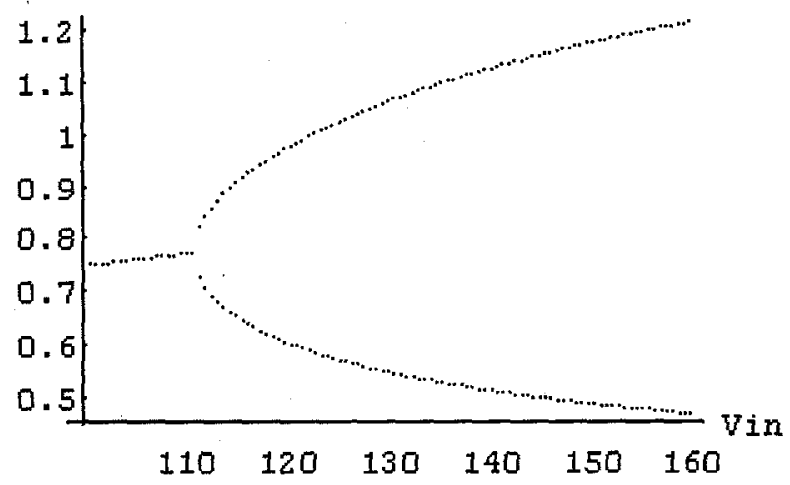

Fig. 11: Evolution of duty cycle for the period-2 orbit

\section{Eigenvalues}

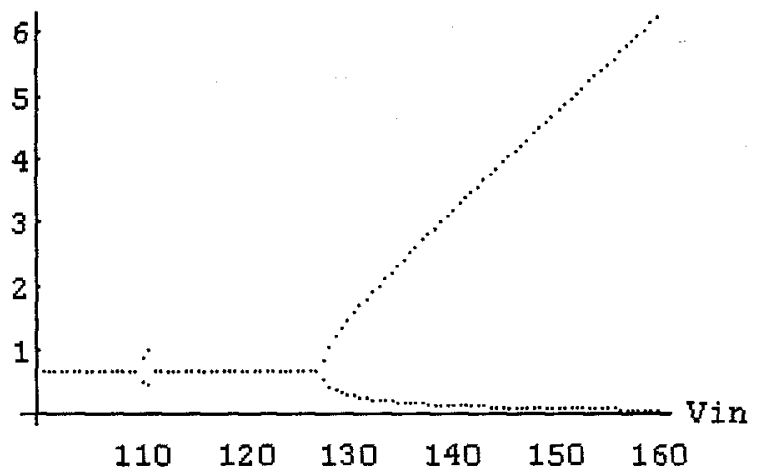

Fig. 12: Evolution of characteristic multipliers for the period-2 orbit

\section{Experimental Results}

In order to further verify the theoretical results, an experimental drive system is prototyped as shown in Fig. 13. The system consists of two identical PM dc servo-motors, namely $M_{1}$ and $M_{2}$, which are directly coupled together. $M_{1}$ acts as the motor which is fed by a buck-type dc chopper, while $\mathrm{M}_{2}$ act as the generator load which is controlled by a Darlington power transistor operating in the linear mode. The built-in tachogenerator in $M_{1}$ is used to provide the speed feedback. The mechanical load torque can be represented by $T_{l}=K_{T} i_{2}+T_{s}$, where $i_{2}$ is the controlled armature current in $\mathrm{M}_{2}$ and $T_{s}$ is the constant friction torque.

The power stage consists of a power MOSFET J162 and a fast-recovery diode U850, while the electronic controller mainly comprises of two operational amplifiers LM833 and a comparator LM311. The LM833 possesses high-frequency performance to handle the wide-bandwidth speed feedback during chaotic operation. The ramp voltage is directly generated by using a function generator.

The switching frequency is selected as low as $250 \mathrm{~Hz}$ so that the parasitic effects can be safely neglected, and the piecewise switched model can accurately describe the drive system. Thus, a filter inductor is connected in series with the armature of $M_{1}$ to ensure the system operating in continuous conduction mode.
The component and parameter values of the this drive system are as follows: $v_{r}=0 \mathrm{~V}, v_{u}=2.2 \mathrm{~V}, T=4 \mathrm{~ms}, g=2, V_{i n}=100 \mathrm{~V}$, $R=3.5 \Omega, \quad L=36 \mathrm{mH}, \quad K_{E}=0.1356 \mathrm{~V} / \mathrm{rads}^{-1}, \quad K_{T}=0.1324 \mathrm{Nm} / \mathrm{A}$, $B=0.000564 \mathrm{Nm} / \mathrm{rads}^{-1}, \quad J=0.000971 \mathrm{Nm} / \mathrm{rads}^{-2}, \quad T_{F}=0.39 \mathrm{Nm}$, $\omega_{\text {ref }}=100 \mathrm{rads}^{-1}$.

By selecting $g=1.5$, the measured waveforms of $v_{c o}$ and $v_{\text {ramp }}$ shown in Fig. 14 illustrate that the system operates in the period-1 orbit, which closely agree with the PSpice simulation waveforms shown in Fig. 7. Then, by increasing $g$, the measured waveforms of $i$ and $v_{c o}$ shown in Fig. 15 illustrate that the system operates in the period- 8 orbit, a subharmonic frequency. By further increasing $g$, chaos occurs. The corresponding measured waveforms of $v_{c o}$ and $v_{\text {ramp }}$ as well as the waveform of $i$ are shown in Figs. 16 and 17, respectively.

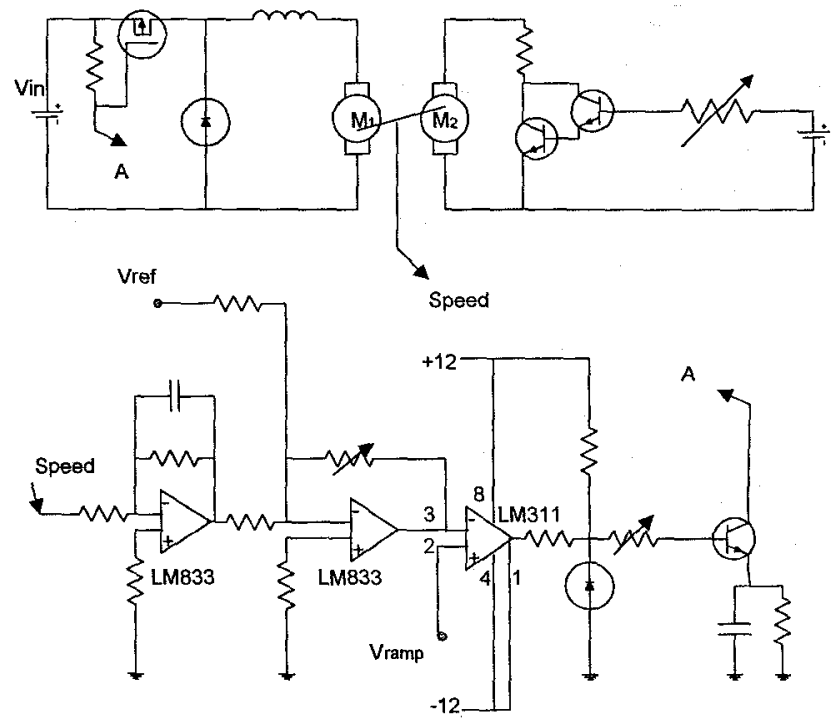

Fig. 13: Schematic diagram of the experimental drive system

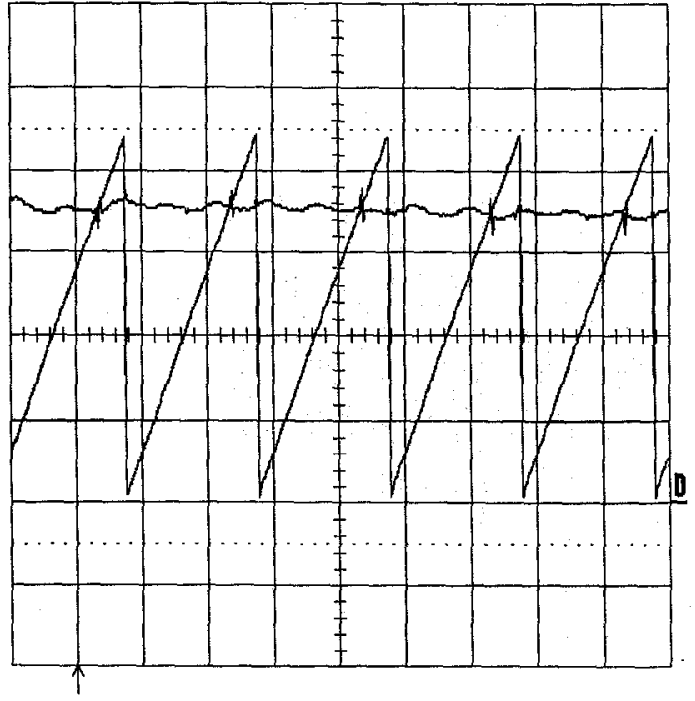

Fig. 14: Measured period-1 waveforms of control voltage and ramp voltage 


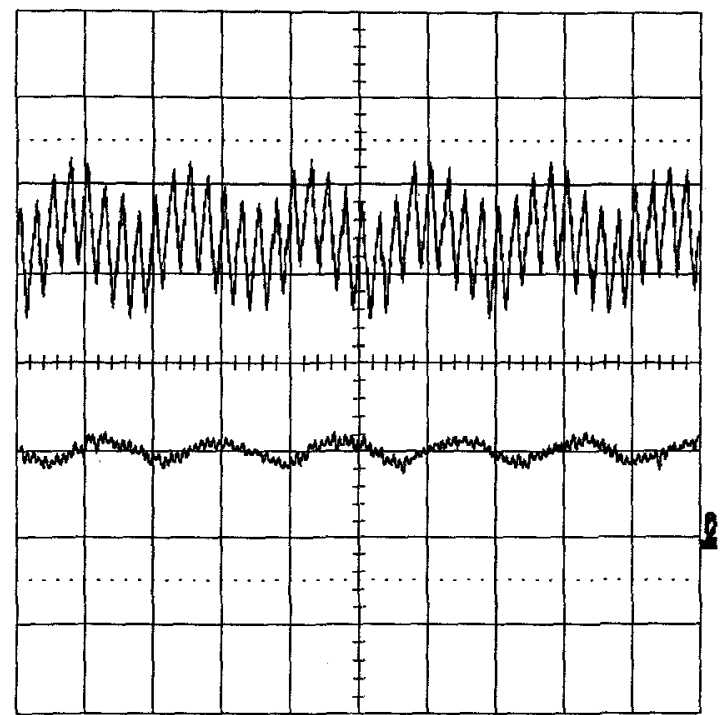

7ig. 15: Measured subharmonic waveforms of armature current and control voltage

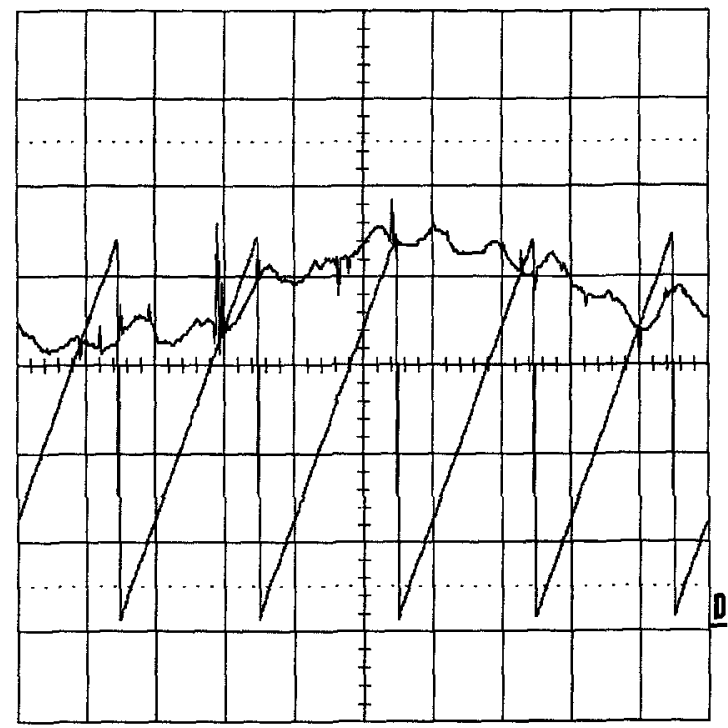

Fig. 16: Measured chaotic waveforms of control voltage and ramp voltage

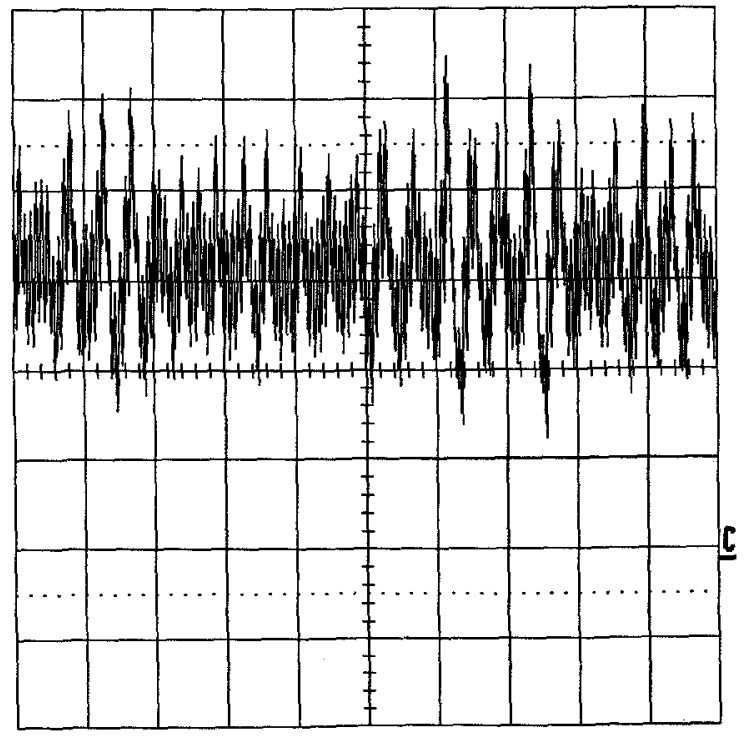

Fig. 17: Measured chaotic waveform of armature current

\section{Conclusion}

In this paper, a second-order generalized iterative map that describes the nonlinear dynamics of the drive system operating in the continuous condition mode has been derived. Based on the derived map, computer simulation reveals that the system not only exhibits a typical period-doubling route to chaos but also the period-3 window. Analytical modeling of the period-1 and period- $p$ orbits that cross the voltage ramp once per cycle, as well as their stability analysis based on evaluating the characteristic multipliers have also been presented. These can greatly facilitate the identification of the desired stable operating ranges during different system parameters and conditions. The theoretical results have been verified by comparing with the bifurcation points occurred in PSpice simulation as well as the measured period-1, subharmonic and chaotic waveforms of the prototype.

Although the investigation has been focused on a typical dc drive, the proposed approach and derived equations can readily be applied or extended to other dc drives.

\section{Acknowledgment}

This work was supported and funded in part by the Committee on Research and Conference Grants, the University of Hong Kong.

\section{References}

[1] D.C. Hamill and D.J. Jefferies, "Subharmonics and chaos in a controlled switched-mode power converter", IEEE Transactions on Circuits and Systems, vol. 35, August 1988, pp.1059-1061

[2] J.H.B. Deane and D.C. Hamill, "Instability, subharmonics, and chaos in power electronic systems", IEEE Transactions on Power Electronics, vol. 5, July 1990, pp.260-268

[3] D.C. Hamill, J.H.B. Dean, and D.J. Jefferies, "Modeling of chaotic DC-DC converters by iterated nonlinear mappings", IEEE Transactions on Power Electronics, vol. 7, January 1992, pp.25-36

[4] E. Fossas and G. Olivar, "Study of chaos in the buck converter", IEEE Transactions on Circuits and Systems-I: Fundamental Theory and Applications, Vol. 43, January 1996, pp.13-25

[5] K. Chakrabarty, G. Poddar, and S. Banerjee, "Bifurcation behavior of the buck converter", IEEE Transactions on Power Electronics, Vol. 11, May 1996, pp.439-447

[6] I. Nagy, L. Matakas Jr. and E. Masada, "Application of the theory of chaos in PWM technique of induction motors", International Power Electronics Conference, Yokohama, 1995, pp.58-63

[7] N. Hemati, "Strange attractors in brushless DC motors", IEEE Transactions on Circuits and Systems-I: Fundamental Theory and Applications, Vol. 41 , January 1994, pp.40-45

[8] T.S. Parker and L.O. Chua, Practical Numerical Algorithm for Chaotic Systems, New York; SpringerVerlag, 1989 